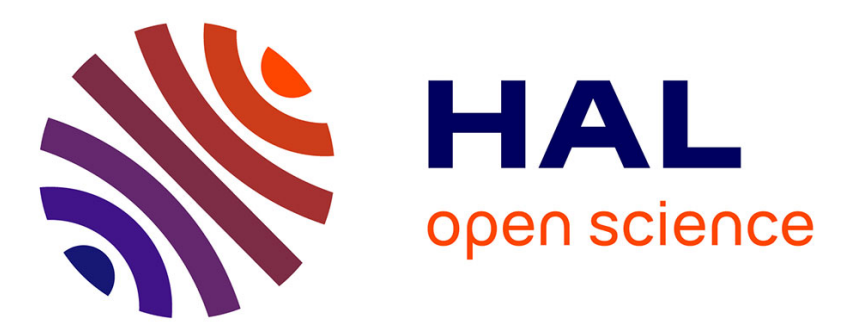

\title{
Cost-effective G-CSF therapy strategies for cyclical neutropenia: mathematical modelling based hypotheses.
}

Catherine Foley, Samuel Bernard, Michael C Mackey

\section{To cite this version:}

Catherine Foley, Samuel Bernard, Michael C Mackey. Cost-effective G-CSF therapy strategies for cyclical neutropenia: mathematical modelling based hypotheses.. Journal of Theoretical Biology, 2006, 238 (4), pp.754-63. 10.1016/j.jtbi.2005.06.021 . hal-00542529

\section{HAL Id: hal-00542529 \\ https://hal.science/hal-00542529}

Submitted on 14 Jan 2020

HAL is a multi-disciplinary open access archive for the deposit and dissemination of scientific research documents, whether they are published or not. The documents may come from teaching and research institutions in France or abroad, or from public or private research centers.
L'archive ouverte pluridisciplinaire HAL, est destinée au dépôt et à la diffusion de documents scientifiques de niveau recherche, publiés ou non, émanant des établissements d'enseignement et de recherche français ou étrangers, des laboratoires publics ou privés. 


\title{
Cost-effective G-CSF therapy strategies for cyclical neutropenia: mathematical modelling based hypotheses
}

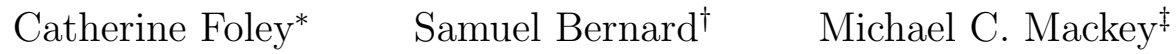

January 9, 2005

\section{Summary}

Using computer simulations of a previously published mathematical model for the regulation of stem cell and neutrophil production in dogs, we have studied the efficacy of three different treatment protocols for cyclical neutropenia involving granulocyte colony stimulating factor (G-CSF). We used a model based on canine data to infer treatments for humans because of the availability of extensive dog data and the similarity of the dog and human disorders. The first treatment scheme involves alternate day administration of G-CSF. The second triggers G-CSF administration whenever neutrophil levels fall below a predetermined level, and the third one follows a random administration protocol. The computer simulations predict that clinically desirable results can be achieved with all three methods, using far less GCSF than would be needed with the standard daily treatment. If the results of this modelling are borne out clinically, it would entail a considerable financial savings to patients.

Keywords: cyclical neutropenia, G-CSF treatment, mathematical modelling, non-standard treatment.

\section{Introduction}

Cyclical neutropenia $(\mathrm{CN})$ is a rare haematological disease characterized by oscillations in the circulating neutrophil count. These levels fall from normal to barely detectable levels with a typical period of 19

*Correspondence: Catherine Foley, Department of Mathematics and Centre for Nonlinear Dynamics, McGill University, 3655 Promenade Sir William Osler, Montreal, QC, CANADA, H3G 1Y6. e-mail: foley@math.mcgill.ca

†Institute for Theoretical Biology, Humboldt University, Invalidenstr. 43, 10115, Berlin, GERMANY. e-mail: s.bernard@biologie.hu-berlin.de

${ }^{\ddagger}$ Departments of Physiology, Physics \& Mathematics and Centre for Nonlinear Dynamics, McGill University, 3655 Promenade Sir William Osler, Montreal, QC, CANADA, H3G 1Y6. e-mail: mackey@cnd.mcgill.ca to 21 days in humans (Guerry et al, 1973; Dale and Hammond, 1988; Haurie et al, 1998), even though periods up to 40 days have been observed (Haurie et al, 1998). These oscillations in the neutrophil count are generally accompanied by oscillations around normal levels in other blood cell lineages such as platelets, lymphocytes and reticulocytes (Haurie et al, 1998, 2000). All grey collies (Lund et al, 1967) are born with this congenital disease and oscillations with period on the order of 11 to 16 days are observed (Haurie et al, 1998, 1999b, 2000). This animal model has provided extensive experimental data to decipher the nature of $\mathrm{CN}$.

Many mathematical models have been proposed to explain the origin of these oscillations as well as to understand the control of neutrophil production in non-pathological cases. For a discussion of previous models that have been developed see Hearn et al (1998) and Haurie et al (1998). In a recent study (Bernard et al, 2003), it was shown that all of the characteristics of $\mathrm{CN}$ in grey collie can be accounted for by an elevated level of cellular death (apoptosis) in the neutrophil precursors. This elevation of apoptosis in $\mathrm{CN}$ was first predicted over twenty years ago (Mackey, 1978) and has been observed clinically (Aprikyan et al, 2001). The modelling predicts that decreasing the level of apoptosis in neutrophil precursors can attenuate or eliminate many of the symptoms of $\mathrm{CN}$.

$\mathrm{CN}$ is often treated using granulocyte colony stimulating factor (G-CSF) (Hammond et al, 1989) which is known to interfere with apoptosis (Koury, 1992; Park, 1996; Williams et al, 1990; Williams and Smith, 1993). Treatment protocols (Amer. Soc. Clin. Oncol., 1997) typically call for daily subcutaneous injection of G-CSF at $5 \mu \mathrm{g}$ per $\mathrm{kg}$ of body weight, and for a $60 \mathrm{~kg}$ adult this currently entails a yearly cost of approximately US $\$ 40,000$ (CF: Check D.C. Dale reference for other dosage AM.J Hematol. 2003). Clearly it would be of enormous help if the same clinical effects could be achieved with the use of less G-CSF. A few alternative treatment strate- 
gies have been reported in which various administration schemes have been used (Jayabose and Sandoval, 1994; Dicato et al, 1992; Danielson and Harmenberg, 1992), yet no attempt to use current knowledge of the dynamic behaviour of the haematopoietic system for designing new treatment protocols has apparently been made.

This paper uses the recent model of Bernard et al (2003) to examine, through computer simulations, the predicted effects of different protocols for the administration of G-CSF to grey collies. The goal is to see whether one can find ways of administering GCSF less frequently than daily and still achieve the same clinical effect currently reached using a daily regimen. In the first section, we describe the model for cyclical neutropenia and its dynamic characteristics. Second, we illustrate the computer predicted results of three different schemes for the administration of G-CSF. The first involves giving G-CSF every other day. The second is a "threshold triggered decision" in which G-CSF is administered whenever the neutrophil count falls below a predetermined value. Finally, the third scheme is a random delivery protocol. The paper concludes with a brief discussion.

\section{The Model}

The model used in this paper considers two cell types whose dynamics are regulated by negative feedback loops. These cell types are the haematopoietic stem cells (HSCs) and the mature neutrophils. HSCs are assumed to be self-renewing and multi-potential. That is, HSCs are self-sustained and newly divided cells can either remain in the HSC pool, or be triggered to undergo differentiation into any of the blood cell lineages.

Fig 1. illustrates the two components of this model: the HSC compartment and the mature neutrophil compartment. The rate at which HSCs differentiate into the neutrophil lineage is assumed to be determined by the blood G-CSF level. As these neutrophil precursors differentiate, their number is amplified through successive divisions.

As shown in Fig 1., there are two feedback loops. The first loop, between the mature neutrophil compartment and the HSC compartment, is the rate $(F(N))$ of HSC differentiation into the neutrophil lineage. $F(N)$ is mediated by G-CSF levels and operates with a lag of duration $\tau_{N}$ that accounts for the time required for neutrophil precursor division and maturation.

The second feedback loop regulates the rate $(K(S))$ at which HSCs enter the proliferative cycle from

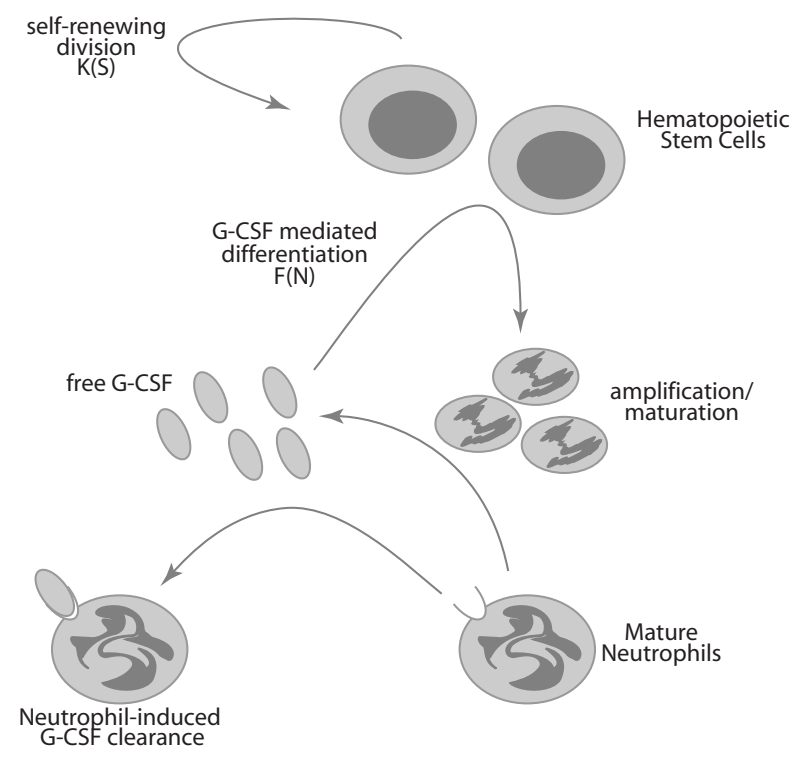

Fig 1: G-CSF mediated neutrophil differentiation.

the resting $\left(\mathrm{G}_{0}\right)$ state. It operates with a delay $\tau_{S}$ that accounts for the cell cycle duration, that is the length of time required to produce two daughter HSCs from one mother cell. $K(S)$ regulates the level of haematopoietic stem cells, while $F(N)$ controls the neutrophil count.

G-CSF acts on model parameters in various ways. First, it decreases the apoptosis rate of neutrophil precursors and HSCs, leading to an increase of HSC levels and neutrophil amplification (Basu et al, 2002). Second, it increases the differentiation rate $F$ of HSC into the neutrophil precursor lineage. Third, the HSC cell cycle duration and the neutrophil precursor transit time are decreased. G-CSF is partly cleared through binding to neutrophil receptors. Thus, GCSF clearance decreases as the number of neutrophils decreases (Kearns et al, 1993; Terashi et al, 1999) and the neutrophil count increases when the level of GCSF is increased (Petros, 1992; Chatta et al, 1994; Price et al, 1996). This type of regulation regulation is a negative feedback mechanism. A similar mechanism is assumed to be in place to control HSC levels, and we take $\mathrm{K}(\mathrm{S})$ to be also a negative feedback loop.

In the context of the mathematical modelling, this interaction has been kept as simple as possible to make model-based predictions possible. The equations of the model are presented in Appendix A.

\section{Behaviour of the model}

The model predicts that for elevated apoptosis rates, oscillations in both the HSC and neutrophil popula- 
tions appear (Bernard et al, 2003). More interestingly, it was shown that for wide parameter range, the system displays bistability. In this case, depending on the history of neutrophil and HSC levels, the neutrophil count may either oscillate or stabilize to a steady state. This bistability phenomenon suggests a potential neutrophil count or phase-dependent treatment on neutrophil levels. Although there is no experimental evidence that bistability really occurs in CN patients, some experimental records (Haurie et $a l, 1999 \mathrm{~b})$ display different responses to G-CSF treatment applied at different times.

Fig 2. shows the absolute neutrophil count (ANC) from a grey collie over 712 days. Two phases of continuous G-CSF treatment were applied (day 0 to day 96 and day 296 to day 436). The two phases without treatment show significant differences in the amplitude of oscillation of ANC and periodicities. Spectral analysis using the Lomb periodogram (a generalization of Fourier spectral analysis for unevenly sampled data) has proven to be useful for analysis of periodic haematological diseases (Fortin and Mackey, 1999; Haurie et al, 1999a). We applied the Lomb periodogram technique to the two phases without treatment. Phase 1 (day 96 to 296) has a period of 14.6 days with strong significance $p \leq 10^{-5}$. Phase 2 also shows some periodicity to the eye (period 15.4 days) but it is not significant. This clear change in the neutrophil dynamics may indicate that the second G-CSF treatment stabilized the neutrophil level, whereas the first phase of treatment did not. Such bistability, if true, would suggest that the neutrophil count can either display periodic fluctuations with large amplitude or stabilize around an average value. In this paper, based on computer simulations we show how the timing of the treatment affects the behaviour of the ANC.

\section{Investigating administration patterning}

Daily G-CSF treatment generally results in a marked increase in average neutrophil count and an elevation in the nadir counts, decreasing the duration of severe neutropenia. An absolute neutrophil count (ANC) below $0.5 \times 10^{9}$ cell $/ \mathrm{L}$ usually defines neutropenia. In investigating different patterning of G-CSF administration with this mathematical model, the number of days of neutropenia in a cycle serve as a benchmark for evaluating efficiency of different treatment strategies.

The mathematical model describes 2 dynamical variables and contains 11 physiological parameters, including 2 time delays. The detailed description of the model is presented in Appendix A. By varying
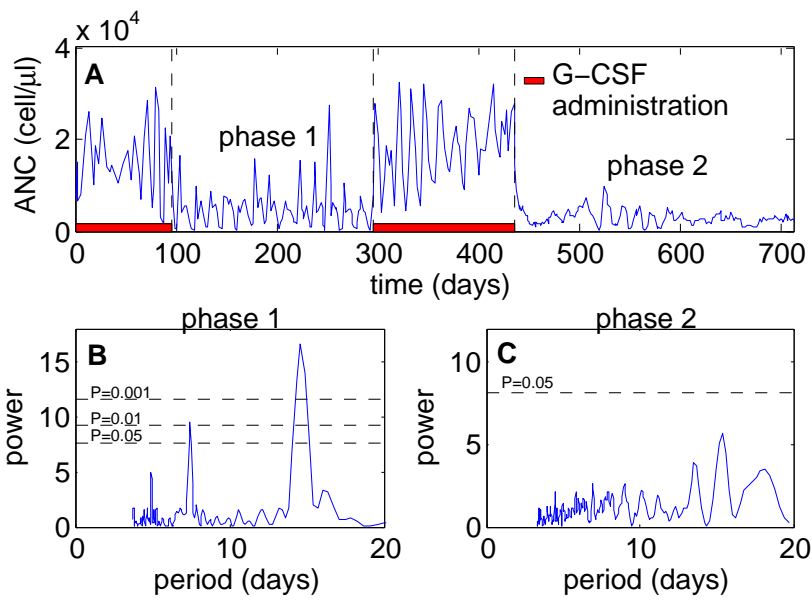

Fig 2: (A) Absolute neutrophil count from a grey collie (Oprah, data generously supplied by Prof. David C. Dale). The red bars show periods of continuous G-CSF treatment. Notice the periodicity in region 1 versus region 2. (B) and (C) show the corresponding Lomb periodograms of phases 1 and 2 respectively.

some of the parameters, we can simulate the effects of G-CSF administration in CN. In Appendix B, we explain how we mimicked the administration of G$\mathrm{CSF}$ as well as the numerical methods that have been used.

\section{The effects of non-standard G- CSF delivery protocols}

To study the effects of non-standard delivery protocols for G-CSF, we compared a daily administration protocol with alternate day, neutrophil-leveldependent and random administration protocols. For each scheme, computer simulations were performed over 70 days ( 5 cycles of 14 days). In each case, we computed the solution for every starting day in the cycle (14 runs for each treatment scheme). The average number of neutropenic days as well as the average number of days of treatment are reported in Table I.

Daily G-CSF administration is generally associated with an increase of the amplitude and a decrease of the period of the oscillations (Haurie et al (1999a)). However, it has also been reported that in some isolated cases G-CSF therapy abolished significant oscillations (Haurie et al (1999a), Hammond et al (1989)). Computer simulations of the model predict that daily administration of G-CSF could either lead to large amplitude oscillations with reduced period of neutropenia, or stabilization of the ANC above the neutropenic level (average of $1.45 \times 10^{9} \mathrm{cell} / \mathrm{L}$, see Fig 3.). We found that the type of response depends on the 


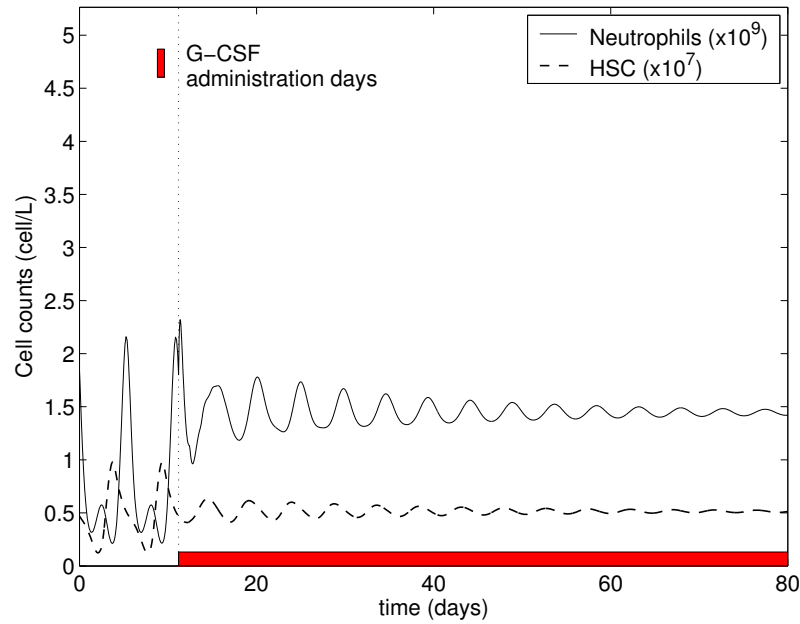

Fig 3: Stabilization of an oscillatory neutrophil count when starting the daily treatment right after the beginning of the falling phase of oscillation.

neutrophil level on the first day of treatment. This suggests that the starting date of the treatment will affect the behaviour of the neutrophil count. For daily and alternate day therapies, this bistability can be characterized in terms of starting day of the treatment (see Fig 4.) and both behaviours (stabilization and oscillations) are distinguished in Table I. The treatment would be optimal when the mathematical model predicts a stabilization of the ANC, as shown in Fig 4. From our numerical simulations, if day 0 represents the day in the cycle at which the ANC nadir occurs, the optimal phase for starting the treatment is between day 3 and day 8 for a cycle length of 14 days. Moreover, the model predicts that starting the treatment during the neutropenic phase (days $0-1$ and days $12-13$ ) will lead to large oscillations.

\section{Alternate day delivery}

In the every-other-day therapy, the model predicts that the blood neutrophil level when G-CSF administration starts (and hence, the starting day of the treatment) also has an effect on the behaviour of the neutrophils. However, because the treatment is interrupted every two days, the behaviour of the neutrophil count is influenced by the ANC every time G-CSF is injected. Consequently, a more erratic response results, but having the same general behaviour, and we can still distinguish a stabilization and an oscillatory phase (see Fig 4 . and Fig 5 . which shows a predicted stabilization behaviour following the cessation of treatment). In this case, the solution is said to be in the stabilization phase if the

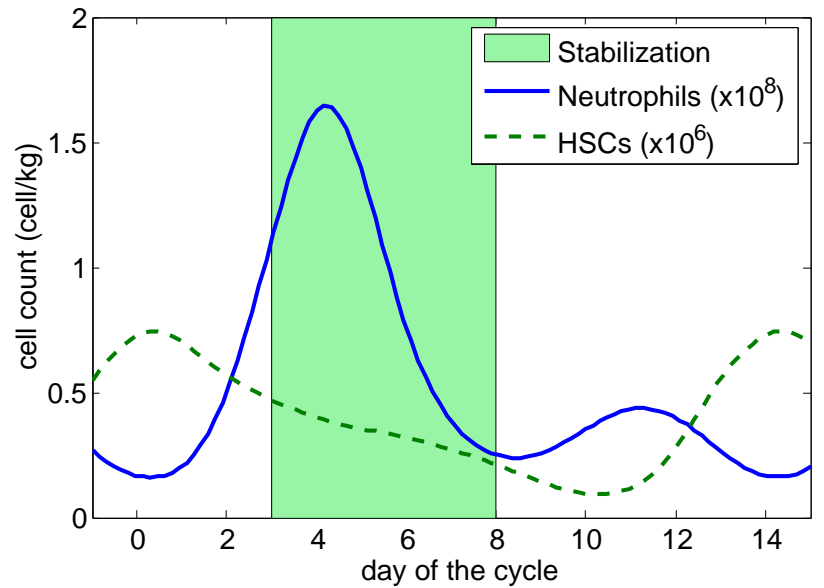

Fig 4: Effect of starting day of treatment for cycles with a period of 14 days using the daily and alternate day schemes. Ideal starting day of treatment (in the stabilization phase) is between day 3 and day 8 .

ANC remains between 0.7 and $2.3 \times 10^{9}$ cells $/ \mathrm{L}$. The range of ideal starting days is as broad as for daily GCSF administration, and this scheme also efficiently increases the nadir of the neutrophil level and shortens the period of neutropenia. Therefore, it seems to achieve positive results similar to daily G-CSF. In particular, one can see from Table I that the results for alternate-day treatment during the stabilization phase are better than the results for the oscillating period of the daily scheme. This suggests that if one is able to localize the stabilization phase for a subject, then starting every-other-day treatment in this period would have similarly good results as continuous G-CSF administration in the oscillatory phase. And more importantly, it uses half the amount of GCSF.

\section{Threshold triggered delivery}

To determine the effects of threshold triggering drug delivery, we simulated G-CSF administration whenever the ANC falls below a predetermined level. Results for levels $0.7,0.9$ and $1.2 \times 10^{9} \mathrm{cell} / \mathrm{L}$ are shown in Table I. The bistability in the parameter space was still present in the results, but there was no obvious distinction between a stabilization and oscillatory phase as a function of starting day of treatment, as for the alternate day and daily schemes (see Fig 6.). This protocol targets specific days when G-CSF is needed and, as a result, it seems that its efficiency is good with respect to both number of neutropenic days and number of treatment days, especially for high predetermined levels (see for instance level $1.2 \times 10^{9} \mathrm{cell} / \mathrm{L}$ in Table I). Although this scheme presents very in- 


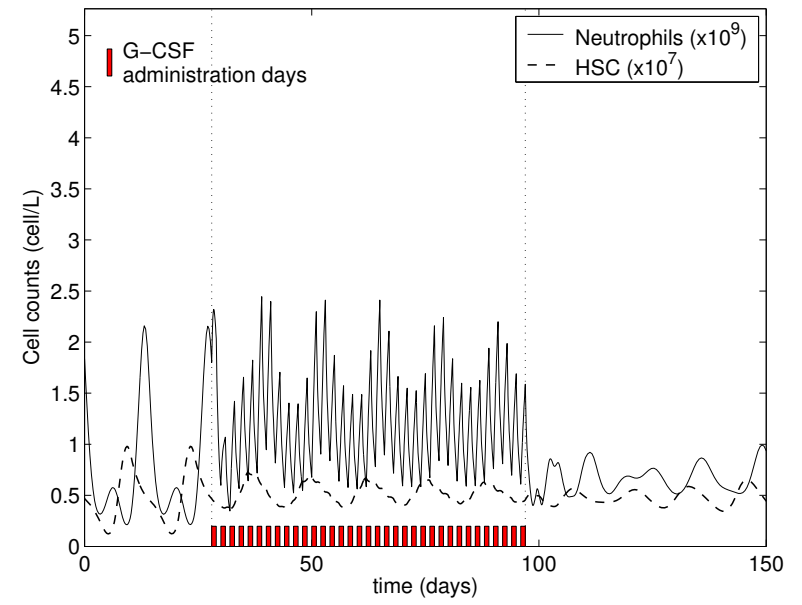

Fig 5: Simulation of the ANC and HSC count using an alternate day regimen, representing a stabilization (the ANC remains between 0.7 and $2.3 \times 10^{9}$ cells/L). Note the low level neutrophil oscillations following the cessation of treatment.

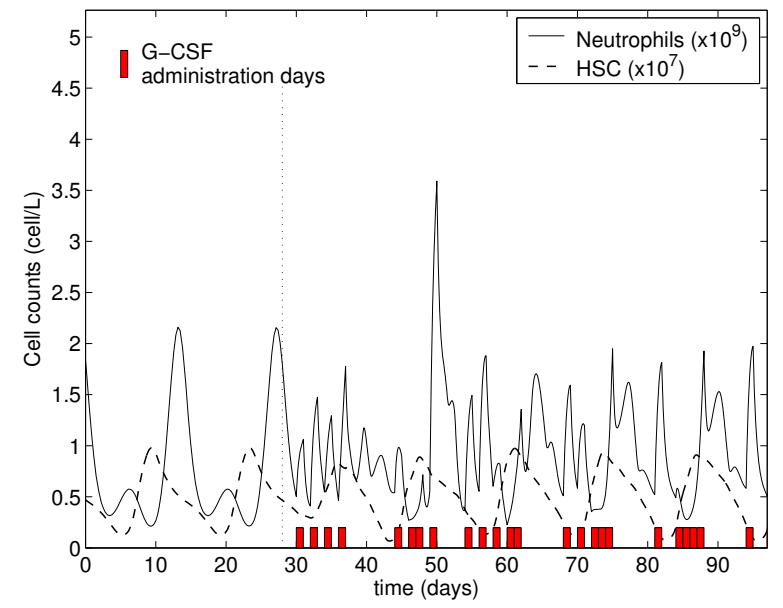

Fig 6: Simulation of the ANC and HSC count using a neutrophil level dependent regimen (threshold level = $\left.0.7 \times 10^{9} \mathrm{cell} / \mathrm{L}\right)$. Over a treatment period of 70 days, there were $35.7 \%$ days of G-CSF administration and $5.7 \%$ days of neutropenia.

teresting theoretical results, it is not a practical approach since it would require monitoring of the blood count frequently and hence, involves expensive laboratory work.

\section{Random protocol}

The results obtained with the level dependent scheme justify finding a scheme that would give similar results, but that would be more easily implemented.

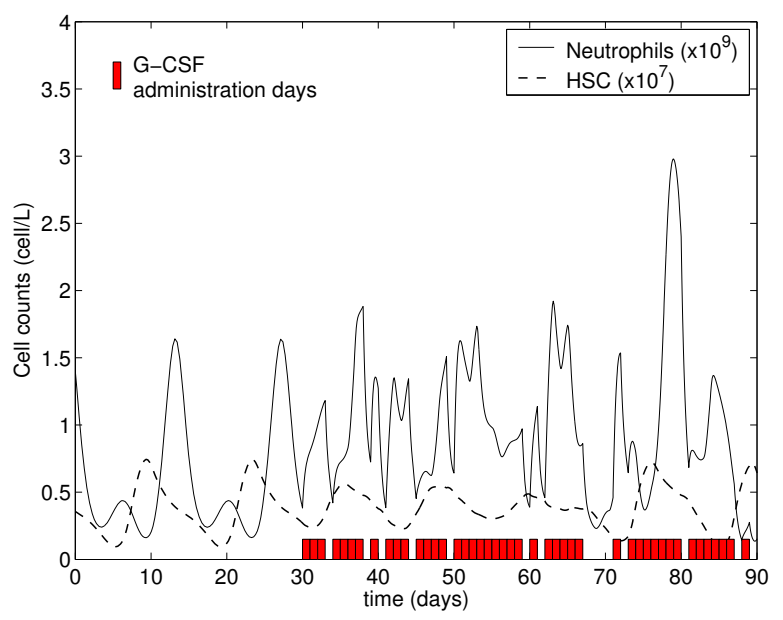

Fig 7: Example of the effects of the random protocol where there were $70 \%$ of treatment days.

We consider a random protocol in which G-CSF is administered randomly for a predetermined number of days. In particular, we use the number of days of administration obtained in the level dependent method as a first indicator (average of $53.8 \%$ of treatment days for level $1.2 \times 10^{9}$ cell/L). However, because the random protocol is obviously not as targeted as the level-dependent scheme, it appears that the number of days of treatment must be higher than for threshold triggered therapy to achieve analogous results. We simulated random administration of G-CSF during 70 days for two cases $(50 \%$ and $70 \%$ of treatment days) (see Fig 7.). Because the random protocol does not necessarily administrate G-CSF the first day of treatment, we only made simulations for starting day 0 and 5 (in the oscillatory and stabilization phase respectively). In each case, we carried out 12 runs and averaged the results, presented in Table I. The protocol with $50 \%$ of treatment days did not give good results. However, the results for the protocol with $70 \%$ of treatment days give similar results as the alternate day therapy. Table I gives a summary of all the methods and their results.

\section{Discussion}

Cyclical neutropenia has been extensively modelled in dogs due to its interesting dynamics and the availability of extensive data for grey collies. However, there have been no attempt to use its dynamic features to improve existing treatment strategies. Using a mathematical model based on canine data, we have provided a computer simulation study of three 


\begin{tabular}{lcc}
\hline \hline Protocol & $\begin{array}{c}\text { Neutr. } \\
\text { days }(\%)\end{array}$ & $\begin{array}{c}\text { Days of } \\
\text { Treatment }(\%)\end{array}$ \\
\hline No Treatment & 34.3 & 0.0 \\
\hline Daily & $3.2(3.4)$ & $100.0(0.0)$ \\
Stabilization & $0.0(0.0)$ & $100.0(0.0)$ \\
Oscillations & $5.6(2.5)$ & $100.0(0.0)$ \\
\hline Alternate Day & $4.4(4.2)$ & $50.0(0.0)$ \\
Stabilization & $0.0(0.0)$ & $50.0(0.0)$ \\
Oscillations & $7.3(2.8)$ & $50.0(0.0)$ \\
\hline Level Dependent & & \\
level =0.7 & $6.3(2.6)$ & $37.9(2.6)$ \\
level =0.9 & $3.0(2.5)$ & $47.6(2.3)$ \\
level =1.2 & $1.1(1.7)$ & $53.8(4.5)$ \\
\hline Random & & \\
50\% Treat. D. & $13.0(7.5)$ & $50.0(0.0)$ \\
70\% Treat. D. & $7.9(4.1)$ & $70.0(0.0)$ \\
\hline \hline
\end{tabular}

Table I: Summary of the different protocols. Values represent means, and standard deviations are in parentheses. For the level dependent protocol, levels are in $\times 10^{9} \mathrm{cell} / \mathrm{L}$.

treatment protocols that achieve essentially the same results as daily administration of G-CSF but with smaller total G-CSF levels and/or fewer days of administration. The first treatment scheme is the alternate day (or every-other-day) regimen, which increases the nadir of the neutrophil level to clinically acceptable levels. The second proposed treatment protocol, neutrophil level-dependent administration, in its current form would be difficult to implement, but an alternative random treatment scheme could easily be designed to achieve similar results. Mimicking the G-CSF administration pattern that was obtained by computer simulation is a possibility that should be considered. However, because there may be some subtle differences between the dog model and the human disease, clinical trials will be necessary to establish efficacy of the treatments in humans.

Moreover, the existence of a region of bistability could be exploited by perturbing the haematopoietic system to stabilize ANC levels. If this were possible, cyclic neutropenia could be triggered into intermittent phases of low level oscillatory, or non-oscillatory, behaviour (c.f. Fig 5. for an example) and only occasional doses of G-CSF would then be required to maintain this state. This idea of using a "physiological black hole" to trigger or destroy oscillations was originally put forward by Winfree $(1973,1980)$ in a different context.

Because of the oscillatory nature of cyclical neutropenia and the nonlinear interactions between GCSF and circulating neutrophils through clearance processes, this disorder is a good candidate for designing dynamic treatment protocols. We have shown, based on a computer simulated model, that phasedependent protocols should be able to achieve equal or better results than standard protocols, while using less G-CSF for fewer days of treatment. Obviously, this result opens up the possibility of decreasing sideeffects as well as increasing the cost-effectiveness of the treatment, thereby improving the quality of life for patients.

\section{References}

Amer. Soc. Clin. Oncol. (1997) 1997 Update of recommendations for the use of hematopoietic colony-stimulating factors: evidence-based, clinical practice guidelines. Journal of Clinical Oncology, 15, 3288.

Aprikyan, A.A.G., Liles, W.C., Rodger, E., Jonas, M., Chi, E.Y., Dale, D.C. (2001) Impaired survival of bone marrow hematopoietic progenitor cells in cyclic neutropenia. Blood, 97, 147-153.

Basu, S., Hodgson, G., Katz, M., Dunn, A.R. (2002) Evaluation of role of g-csf in the production, survival, and release of neutrophils from bone marrow into circulation. Blood, 100, 854-861.

Bernard, S., Bélair, J., Mackey, M.C. (2003) Oscillations in cyclical neutropenia: New evidence based on mathematical modeling. Journal of Theoretical Biology, 223, $283-298$.

Chatta, G.S., Price, T.H., Allen, R.C., Dale, D.C. (1994) Effects of in vivo recombinant methionyl human granulocyte colony stimulating factor on the neutrophil response and peripheral blood colony forming cells in healthy young and elderly adult volunteers. Blood, 84, 2923-2929.

Dale, D.C., Hammond, W.P. (1988) Cyclic neutropenia: A clinical review. Blood Review, 2, 178-185.

Danielson, L., Harmenberg, J. (1992) Intermittent g-csf in cyclic neutropenia. European Journal of Hematology, 48, 123-124.

Dicato, M., Meyer, S., Ries, F. (1992) Reduced G-CSF dosage in cyclic neutropenia gives satisfactory clinical laboratory and economic results. Blood, 80, 413.

Fortin, P., Mackey, M.C. (1999) Periodic chronic myelogenous leukemia: Spectral analysis of blood cell counts and etiological implications. British Journal of Haematology, 104, 336-345.

Guerry, D., Dale, D.C., Omine, M., Perry, S., Wolff, S.M. (1973) Periodic hematopoiesis in human cyclic neutropenia. Journal of Clinical Investigation, 52, 32203230 . 
Hammond, W.P., Price, T.H., Souza, L.M., Dale, D.C. (1989) Treatment of cyclic neutropenia with granulocyte colony stimulating factor. New England Journal of Medicine, 320, 1306-1311.

Haurie, C., Dale, D.C., Mackey, M.C. (1998) Cyclical neutropenia and other periodic hematological diseases: A review of mechanisms and mathematical models. Blood, 92, 2629-2640.

Haurie, C., Dale, D.C., Mackey, M.C. (1999a) Occurrence of periodic oscillations in the differential blood counts of congenital, idiopathic and cyclical neutropenic patients before and during treatment with G-CSF. Experimental Hematology, 27, 401-409.

Haurie, C., Dale, D.C., Rudnicki, R., Mackey, M.C. (2000) Modeling complex neutrophil dynamics in the grey collie. Journal of Theoretical Biology, 204, 505519 .

Haurie, C., Person, R., Dale, D.C., Mackey, M.C. (1999b) Haematopoietic dynamics in grey collies. Experimental Hematology, 27, 1139-1148.

Hearn, T., Haurie, C., Mackey, M.C. (1998) Cyclical neutropenia and the peripheral control of white blood cell production. Journal of Theoretical Biology, 192, 167181.

Jayabose, S., Sandoval, C. (1994) Recombinant human granulocyte colony stimulating factor in cyclic neutropenia: Use of a new 3-day-a-week regimen. The American Journal of Pediatric Hematology/Oncology, 16, 338-340.

Kearns, C.M., Wang, W.C., Stute, N., Ihle, J.N., Evans, W.E. (1993) Disposition of recombinant human granulocyte colony stimulating factor in children with severe chronic neutropenia. Journal of Pediatrics, 123, 471479 .

Koury, M.J. (1992) Programmed cell death (apoptosis) in hematopoiesis. Experimental Hematology, 20, 391-394.

Lund, J.E., Padgett, G.A., Ott, R.L. (1967) Cyclic neutropenia in grey collie dogs. Blood, 29, 452-461.

Mackey, M.C. (1978) Unified hypothesis of the origin of aplastic anemia and periodic hematopoiesis. Blood, 51, 941-956.

Park, J.R. (1996) Cytokine regulation of apoptosis in hematopoietic precursor cells. Current Opinion in Hematology, 3, 191-196.

Petros, W.P. (1992) Pharmacokinetics and administration of colony-stimulating factors. Pharmacotherapy, 12, 32S-38S.

Price, T.H., Chatta, G.S., Dale, D.C. (1996) Effect of recombinant granulocyte colony stimulating factor on neutrophil kinetics in normal young and elderly humans. Blood, 88, 335-340.
Shampine, L.F., Thompson, S. (2001) Solving ddes in, applied numerical mathematics. Applied Numerical Mathematics, 37, 441-445.

Terashi, K., Oka, M., Ohdo, S., Furukubo, T., Ikeda, C., Fukuda, M., Soda, H., Higuchi, S., Kohno, S. (1999) Close association between clearance of recombinant human granulocyte colony-stimulating factor (G$\mathrm{CSF}$ ) and G-CSF receptor on neutrophils in cancer patients. Antimicrobial Agents and Chemotherapy, 43, $21-24$.

Williams, G., Smith, C. (1993) Molecular regulation of apoptosis: Genetic controls on cell death. Cell, 74, 777-779.

Williams, G.T., Smith, C.A., Spooncer, E., Dexter, T.M., Taylor, D.R. (1990) Haemopoietic colony stimulating factors promote cell survival by suppressing apoptosis. Nature, 343, 76-79.

Winfree, A.T. (1973) Time and timelessness in biological clocks. In Urquhart, J., Yates, F.E., eds., Temporal Aspects of Therapeutics, pp. 35-57. Plenum, New York.

Winfree, A.T. (1980) The Geometry of Biological Time. Springer-Verlag, New York, 1st ed.

\section{Acknowledgments}

This work was supported by MITACS (Canada) and the Natural Sciences and Engineering Research Council (NSERC grant OGP-0036920, Canada). We thank Prof. David Dale and Ms. Caroline Haurie for providing the grey collie data.

\section{Appendix A. Mathematical model}

The model for neutrophil production consists of a system of two delay differential equations (DDEs). Each equation considers the net production and loss rates (termed $P(t)$ and $L(t)$ respectively) of haematopoietic stem cells and mature neutrophils.

$$
\begin{aligned}
\frac{d S(t)}{d t} & =P_{S}(t)-L_{S}(t), \\
\frac{d N(t)}{d t} & =P_{N}(t)-L_{N}(t) .
\end{aligned}
$$

The net production rates are:

$$
\begin{aligned}
P_{S}(t) & =2 e^{-\gamma_{S} \tau_{S}} S\left(t-\tau_{S}\right) K\left(S\left(t-\tau_{S}\right)\right) \\
P_{N}(t) & =A S\left(t-\tau_{N}\right) F\left(N\left(t-\tau_{N}\right)\right) .
\end{aligned}
$$


The delayed arguments account for the time required for cell division cycles and maturation to occur. The net loss rates are:

$$
\begin{aligned}
L_{S} & =S(t)[F(N(t))+K(S(t))], \\
L_{N} & =\alpha N(t) .
\end{aligned}
$$

The variable $S$ represents the number of haematopoietic stem cells in the resting $\left(G_{0}\right)$ phase. Cells in the resting phase can either enter the proliferative phase at a rate $K(S)$ or differentiate at a rate $F(N)$ to ultimately give rise to mature neutrophils, represented by the second variable, $N$. Cells in the HSC proliferative phase undergo apoptosis at a rate $\gamma_{S}$ and the cell cycle duration is $\tau_{S}$. The fraction of HSC surviving cell division is then $\exp \left(-\gamma_{S} \tau_{S}\right)$. Successive divisions amplify cell numbers in the differentiation pathway by a factor $A$, which is also used to account for cell loss due to apoptosis. After a time $\tau_{N}$, each differentiated HSC gives rise to $A$ mature neutrophils $N$, which are released into the circulation. It is assumed that mature neutrophils are eliminated at a rate $\alpha$.

Two feedback loops control the entire process through the neutrophil differentiation rate $F(N)$ and the HSC proliferation rate $K(S)$. G-CSF mediates neutrophil differentiation through a clearance mechanism. When neutrophil count decreases, C-CSF level goes up due to the lack of clearance and differentiation increases. As a function of the neutrophil count, the differentiation rate is thus monotonically decreasing (here G-CSF is implicitly taken into account), and is described by a decreasing Hill function,

$$
F(N)=f_{0} \frac{\theta_{1}^{n}}{\theta_{1}^{n}+N^{n}},
$$

where $f_{0}$ is the maximal differentiation rate (i.e. in absence of neutrophil), $n$ is a cooperativity coefficient and $\theta_{1}$ is the neutrophil concentration at which differentiation is half maximal. Notice that $\theta_{1}$ depends on endogenous G-CSF production and G-CSF administration. HSC proliferation is assumed to be regulated through a similar mechanism. When the HSC level is depleted, intercellular signals trigger cell replication. These signals become fainter when HSC level increases back to normal levels. The HSC proliferation rate feedback term is thus also described by a decreasing Hill function,

$$
K(S)=k_{0} \frac{\theta_{2}^{s}}{\theta_{2}^{s}+S^{s}} .
$$

The parameter $k_{0}$ is the maximal proliferation rate, $s$ is a cooperativity coefficient and $\theta_{2}$ the HSC concentration at which HSC proliferation is half maximal. See Bernard et al (2003) for a detailed derivation of the feedback functions.

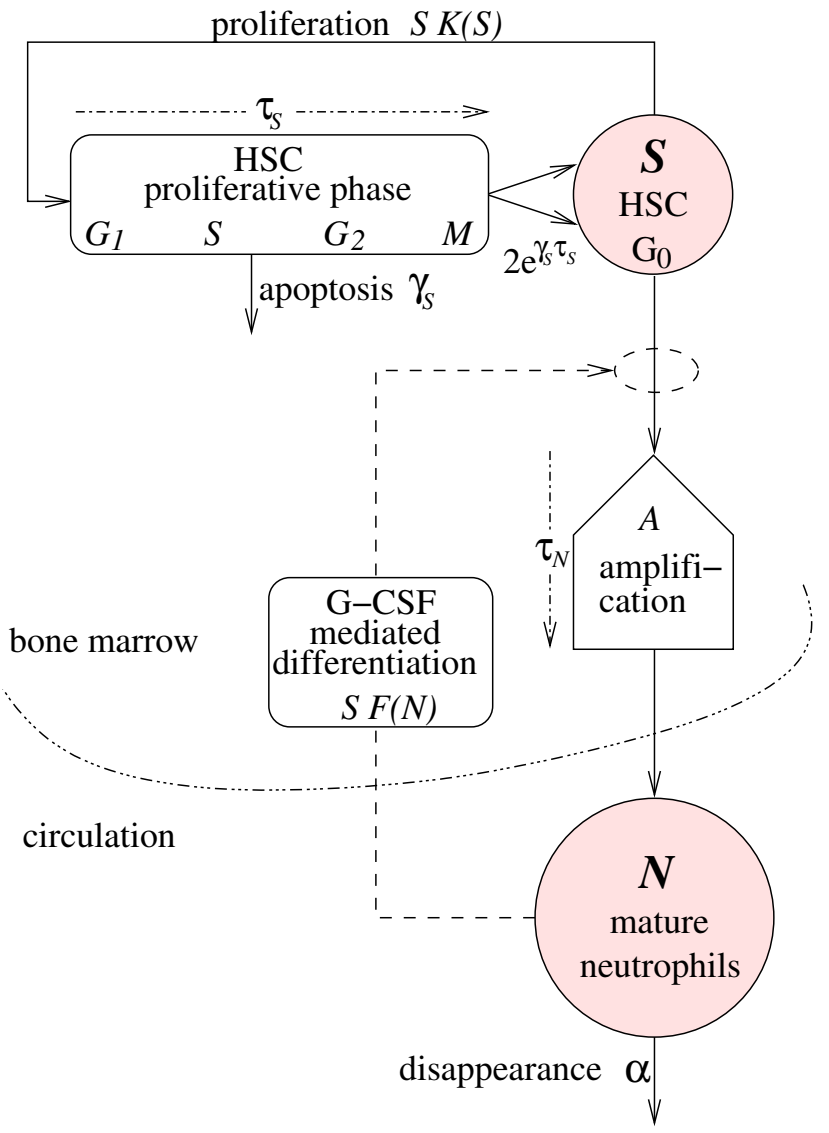

Fig 8: Model of neutrophil production. The variable $S$ represents the number of haematopoietic stem cells in the resting $\left(G_{0}\right)$ phase. Cells in the resting phase can either enter the proliferative phase at a rate $K(S)$ or differentiate at a rate $F(N)$ to ultimately give rise to mature neutrophils $N$, the second variable. Cells in the HSC proliferative phase undergo apoptosis at a rate $\gamma_{S}$ and the cell cycle duration is $\tau_{S}$. Successive divisions amplify cell number in the differentiation pathway by a factor $A$, which is also used to account for cell loss due to apoptosis. After a time $\tau_{N}$, differentiated cells become mature neutrophils $N$ and are released into the blood. It is assumed that mature neutrophils die at a fixed rate $\alpha$. Two feedback loops control the entire process through the proliferation rate $K(S)$ and the differentiation rate $F(N)$.

\section{Appendix B. Simulating CN and G-CSF administration}

Bernard et al (2003) showed that the model can predict cyclical neutropenia and simulate G-CSF administration by using physiologically relevant values for the 11 different parameters. The parameters used for numerical simulation are those presented in Bernard et al (2003) and they are in agreement with experimental data. Table II presents the parameter values 
used for mimicking the neutrophil production in three situations of interest: in a healthy subject (without $\mathrm{CN}$ ), in a dog suffering from CN, and under G-CSF treatment. For more details on the choice and estimation of those parameters, see Bernard et al (2003).

Numerical simulations were performed using the DDE solver dde23 (Shampine and Thompson, 2001) for Matlab, which solves delay differential equations with constant delays. Mimicking G-CSF injection in a cyclic neutropenic subject was done by simply changing the corresponding sets of parameters. Five parameters were modified under the influence of $\mathrm{G}$ CSF: the neutrophil amplification $A$, the HSC apoptosis rate $\gamma_{S}$, the half-differentiation level $\theta_{1}$, the cell cycle duration $\tau_{S}$ and the maturation transit time $\tau_{N}$. We first computed the solution with the CN parameters for a transient period of 200 days. This allowed the system to settle down to the stable periodic solution and gives a good approximation of the behaviour in the CN situation. Then, administration of G-CSF was simulated by solving the system using the set of parameters corresponding to G-CSF. For instance, to simulate the alternate day treatment, we first integrated the DDE system using the CN parameters for one day. Then, we integrated the equations using the G-CSF parameters for the next day, and we kept switching from one parameter set to the other during the desired simulated treatment period. Note that the values of the delays are modified to mimic G-CSF. Because dde23 only allows constant delays, this leads to assumptions on G-CSF kinetics since we could not simulate a gradual decrease in the time delays and the other parameter values when switching from CN to G-CSF. This corresponds to assuming that the effects of G-CSF are immediate when injected, and that those effects last exactly one day before disappearing completely and instantaneously.

\begin{tabular}{|c|c|c|c|c|}
\hline Parameter & Unit & "Health & $\overline{\mathrm{CCN}}$ & 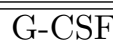 \\
\hline$A$ & 100 & 380 & 10 & 20 \\
\hline$f_{0}$ & day $^{-1}$ & 0.8 & 0.8 & 0.8 \\
\hline$\theta_{1}$ & $10^{8}$ cell $/ \mathrm{kg}$ & 0.36 & 0.36 & 0.8 \\
\hline$k_{0}$ & day $^{-1}$ & 8.0 & 8.0 & 8.0 \\
\hline$\theta_{2}$ & $10^{6}$ cell $/ \mathrm{kg}$ & 0.095 & 0.095 & 0.095 \\
\hline$n$ & - & 1 & 1 & 1 \\
\hline$s$ & - & 2 & 2 & 2 \\
\hline$\tau_{N}$ & day & 3.5 & 3.5 & 3.0 \\
\hline$\tau_{S}$ & day & 2.8 & 2.8 & 2.6 \\
\hline$\gamma_{S}$ & day & 0.07 & 0.07 & 0.05 \\
\hline$\alpha$ & $\mathrm{day}^{-1}$ & 2.4 & 2.4 & 2.4 \\
\hline
\end{tabular}

Table II: Estimated model parameters used for numerical simulations of healthy condition, cyclical neutropenic condition and under G-CSF treatment. Values from Bernard et al (2003). 